\title{
Cálculo mental em questão: fundamentação teórica e reflexões
}

\author{
Keli Cristina Conti \\ Laís Macedo de Almeida Nunes
}

\begin{abstract}
Resumo: Este artigo é resultado de um projeto de Iniciação Cientifica e contempla uma pesquisa bibliográfica na qual foram levantadas informações de artigos e documentos sobre o "cálculo mental". Esta publicação tem como objetivo entrelaçar diferentes aspectos teóricos sobre essa temática. Foram exploradas a definição de cálculo mental, bem como estratégias para desenvolvê-lo. Além disso, são apresentadas vantagens que essa prática pode oferecer aos estudantes. A partir do estudo, pudemos refletir sobre a importância de se trabalhar o cálculo mental em todos os anos da escola básica. Em conclusão, salientamos a relevância dessa temática ser trabalhada não só com estudantes, mas também na formação de professores, para que esses possam refletir sobre essa modalidade de cálculo e, consequentemente, incentivar sua prática em sala de aula.
\end{abstract}

Palavras-chave: Educação Matemática. Cálculo Mental. Ensino de Matemática. Escola Básica.

\section{Mental calculation in question: theoretical basis and reflections}

Abstract: This article is the result of a Junior Researcher Program that collected bibliographical research data surveying information in articles and documents related to "mental calculation". The objective of this article is to interweave different aspects of this subject, according to the perspective of several authors. It explored the definition of mental calculation as well as strategies to develop it. In addition, different advantages that this practice can offer students have been presented. From this study, we could reflect on

Keli Cristina Conti iD Doutora em Educação (UNICAMP). Professora do Mestrado Profissional em Educação da Universidade Federal de Minas Gerais. Minas Gerais, Brasil. $\$ keli.conti@gmail.com

Laís Macedo de Almeida Nunes Aluna da Licenciatura em Matemática da Universidade Federal de Minas Gerais. Minas Gerais, Brasil. $\bowtie$ laismanunes@gmail.com

Recebido em 11/15/2018 Aceito em 08/02/2019 Publicado em 01/09/2019 the importance of using mental calculation in all years of school. In conclusion, we emphasize the relevance of this theme for students and future teachers, so that they can reflect on this modality of calculation and consequently encourage their practice in the classroom.

Keywords: Mathematics Education. Mental Calculation. Mathematics Teaching. Basic school.

\section{Cálculo mental en cuestión: fundamentación teórica y reflexiones}

Resumen: El presente artículo es el resultado de un primer proyecto de iniciación científica, en el que se contempla una investigación bibliográfica de artículos y documentos informaciones sobre el "cálculo mental". Esta publicación tiene como objetivo analizar y entrelazar diferentes aspectos de la temática susodicha, de acuerdo con diferentes autores. Se exploraron la definición de cálculo mental, así como estrategias para desarrollarlo. Además, se presentaron diferentes ventajas que esta práctica puede ofrecer a los estudiantes. A partir del estudio, pudimos reflexionar sobre la importancia de trabajar el cálculo mental durante el transcurso de la primaria o escuela básica. Y a modo de conclusión, subrayó la relevancia de esta temática ser trabajada no sólo con estudiantes, sino también en la formación de docentes, para que puedan reflexionar sobre esa modalidad de cálculo y consecuentemente incentivar su práctica en el aula.

Palabras clave: Educación Matemática. Cálculo Mental. Enseñanza de Matemáticas. Escuela Básica. 


\section{Introdução}

Em meio às atuais propostas de uso de novas tecnologias e calculadoras na sala de aula, surge, também, a necessidade da revalorização do cálculo mental. Revalorização porque, anteriormente ao movimento da Matemática Moderna, essa prática já era valorizada pela sua utilidade e praticidade, e, após o movimento, a utilização do cálculo mental tem sido valorizada novamente tanto pela sua utilidade quanto pelo seu valor cognitivo (GOMES, 2007).

Os Parâmetros Curriculares Nacionais (BRASIL, 1997, 1998) apontam as relações e fundamentações entre os diferentes tipos de cálculo e a importância de se trabalhar, concomitantemente, as suas quatro modalidades, sendo elas o cálculo escrito, as estimativas, 0 cálculo mental e as calculadoras. A atual Base Nacional Comum Curricular (BRASIL, 2017a) também propõe a abordagem do cálculo mental na Escola Básica, no entanto, apenas para os anos iniciais do Ensino Fundamental.

Dessa forma, diferentes pesquisadores têm feito dessa modalidade seu foco de estudo e pesquisa, com o objetivo de destacar as diferentes utilidades e abordagens que o cálculo mental possui. $O$ norte teórico deste artigo partiu das análises realizadas por esses pesquisadores em suas respectivas publicações, tais como Gomes (2007), que apresenta aspectos históricos, como sua abordagem pré e pós Matemática Moderna; Carvalho (2011), que exibe a importância e algumas estratégias para desenvolver o cálculo mental; e Parra (1996), que expõe aspectos teóricos como definição e vantagens de sua prática.

Os estudos citados acima mostram, de início, a relevância do objeto a ser estudado neste artigo para a aprendizagem da Matemática. Este artigo difere dos demais, pois faz se uma revisão, uma coletânea de diferentes e relevantes aspectos teóricos de maneira a entrelaçar as ideias defendidas pelos autores.

Assim sendo, o presente trabalho prossegue apresentado a metodologia da pesquisa para que, em sequência, seja exposto a definição de cálculo mental e, logo em seguida, problematizálo como um dos pilares do cálculo. Posteriormente, é apresentado também as vantagens do cálculo mental, destacando a importância de seu desenvolvimento, seguidas de estratégias e propostas para cada operação matemática. Por fim, serão levantadas considerações em relação à discussão no decorrer do texto. 


\section{Metodologia}

Esta pesquisa de iniciação científica integra um projeto intitulado Contribuições do Laboratório de Ensino de Matemática para a formação inicial do professor que ensina Matemática, que visa ampliar o Laboratório de Ensino de Matemática (LEM) na Faculdade de Educação da Universidade Federal de Minas Gerais (FaE/UFMG). O projeto busca analisar e interpretar práticas de formação e de atuação de futuros professores de forma a compreender e ressaltar a importância de um LEM para a formação inicial do professor que ensinará Matemática e seu reflexo nos processos de ensino e de aprendizagem dos estudantes.

Nesse sentido, a pesquisa se dividiu em duas fases: a primeira foi uma pesquisa bibliográfica, um estudo documental de acordo com Fiorentini e Lorenzato (2006), em que foram feitos levantamentos de livros, publicações, artigos e dissertações que tratavam da temática do cálculo mental, a fim de, inicialmente, aprofundar-se sobre o assunto. Esse levantamento se iniciou após a leitura da publicação de Araújo e Soares (2002), de maneira que se pesquisando a palavrachave "cálculo mental" no Google Acadêmico surgiram outros artigos que também foram lidos. A partir do referencial teórico desses artigos, foi-se encontrando outras publicações interessantes, que abordavam aspectos teóricos e práticos com o cálculo mental.

Além da pesquisa de artigos, também foram consultados documentos brasileiros, como os Parâmetros Curriculares Nacionais (BRASIL, 1997, 1998), a Base Nacional Comum Curricular (2017a) e o Programa Nacional do Livro Didático (2017b, 2018) a fim de analisar como tais documentos abordam e norteiam o estudo e a prática de cálculo mental. Por meio desses artigos e documentos, foi possível encontrar diferentes e importantes referências sobre o assunto, os quais, foram selecionados de maneira a abordar diversas perspectivas sobre o assunto, como por exemplo aspectos teóricos, aspectos históricos, relatos de experiências e entre outros.

A segunda fase da pesquisa foi um reflexo da primeira, e culminou na produção deste artigo, o qual tem como objetivo entrelaçar diferentes aspectos teóricos sobre o cálculo mental, de acordo com a perspectiva de diversos autores. Dessa forma, foram exploradas a definição de cálculo mental, bem como estratégias para desenvolvê-lo, relacionando-as com as propriedades matemáticas. Além disso, são apresentadas algumas vantagens que essa prática pode oferecer aos estudantes. Visto isso, consideramos importante trabalhar essa temática não apenas com os alunos, mas também com os futuros professores de maneira que os incentivem a estimular essa prática em sala de aula 
Apresentado tais fatos, passa-se, com base nos estudos realizados, a se explorar a definição de cálculo mental, para posteriormente ser explicitado, de acordo com o referencial teórico, a relação entre as quatro modalidades de cálculo.

\section{0 que é cálculo mental?}

De acordo com o Dicionário Aurélio, "cálculo" é definido como "fazer cálculos matemáticos; determinar por meio do cálculo", e "mental" é indicado como "que se faz mentalmente". Unindo os dois significados, "cálculo mental" seria qualquer cálculo e conta matemática que se faz mentalmente. Parra (1996) afirma que a expressão "cálculo mental" pode apresentar diferentes significados, dividindo as opiniões dos pesquisadores e ocasionando dúvidas.

A definição de cálculo mental não é única entre os autores estudados, no entanto, eles entram em consenso que cálculo mental é aquele desenvolvido, preferivelmente, com a "cabeça". Dessa forma, Carvalho $(2011$, p. 1) aponta uma pergunta pertinente sobre o que é cálculo mental: seria um cálculo feito de cabeça ou um cálculo feito com a cabeça?

Por mais que seja pequena a diferença, de acordo com as definições dadas por Parra (1996) e Bourdenet (2007), entendemos como cálculo de cabeça um cálculo pensado ou refletido, que utiliza estratégias para ser efetuado. Já cálculo na cabeça, também de acordo com as definições dos autores já referidos, seria um tipo de cálculo automático, um cálculo que efetua a conta armada mentalmente utilizando os algoritmos das operações. Taton (1969) apud Carvalho (2011), enfatiza que o cálculo escrito efetuado de memória, de maneira mental, seria nada mais nada menos que uma forma do cálculo mental adaptado. Parra (1996) ainda afirma que essas duas vertentes do cálculo mental se relacionam, de forma que o cálculo pensado deve acompanhar um aumento progressivo do cálculo automático.

Para alguns, cálculo mental está relacionado à rapidez com que se faz uma conta ou à memorização. Para outros, cálculo mental é o oposto de cálculo escrito, sendo considerado então qualquer cálculo que possa ser feito sem anotações, sem "lápis e papel". Para Taton (1969), citado por Carvalho (2011), como essas duas modalidades de cálculo utilizam um encadeamento de operações mentais elementares, elas são, portanto, semelhantes, não opostas.

De acordo com Parra (1996), cálculo mental são todos os procedimentos que, após a análise de dados, se relacionam e articulam sem a necessidade de recorrer a um algoritmo préestabelecido para obter os resultados aproximados ou exatos. A autora ainda corrobora a ideia de 
que o cálculo mental não exclui a possível utilização de papel e lápis em registros intermediários, pois o processo geral é feito mentalmente.

O significado de cálculo mental apontado pelo Dicionário Enciclopédico Luso-brasileiro VI, publicação de 1991, citado por Carvalho (2011, p. 1) é: "operação aritmética, feita de memória, sem auxílio de sinais escritos". No entanto, assim como Parra (1996), Bourdenet (2007) discorda desse significado, trazendo a seguinte definição:

O que chamaremos de cálculo mental vai além do cálculo mental tradicional ou automatizado, incluiremos o cálculo pensado ou fundamentado, que torna possível reconstruir os cálculos por raciocínios adequados, bem como cálculo mental literal. Os procedimentos serão, portanto, diversos e sua diversidade deverá ser levada em consideração na correção, evitando privilegiar precipitadamente um deles. Dessa forma, insistiremos na importância do método, mais do que na sua rapidez de execução que, no entanto, não deve ser negligenciada. Se necessário, o aluno pode escrever alguns cálculos e resultados intermediários. (BOURDENET, 2007, p. 6, grifos nossos, tradução nossa).

Vista a definição de cálculo mental para alguns autores, é perceptível que outras modalidades do cálculo são, também, citadas. Isso posto, o próximo tópico apresentará os quatro tipos de cálculo, para também explicitar suas relações com o cálculo mental.

\section{Os pilares do cálculo e suas relações com o cálculo mental}

Bigode e Gimenez (2010) enfatizam que, para o desenvolvimento de competências de cálculo, é importante um equilíbrio entre as quatro modalidades de cálculo: cálculo escrito, cálculo mental, estimativa e calculadora. Considerando essas modalidades como os pilares do cálculo, os referidos autores fazem uma analogia com uma mesa de quatro pés: se algum pé da mesa for maior que outro, essa mesa cambaleará, isto é, não estará em equilíbrio, o que só é possível se os pés forem todos iguais. Ou seja, se cada pé da mesa representa um dos pilares do cálculo, eles devem ser todos valorizados e estimulados da mesma forma para que haja um equilíbrio entre as quatro competências. Guimarães e Freitas (2007) consideram que nas escolas brasileiras predomina o uso do cálculo escrito e exato. 


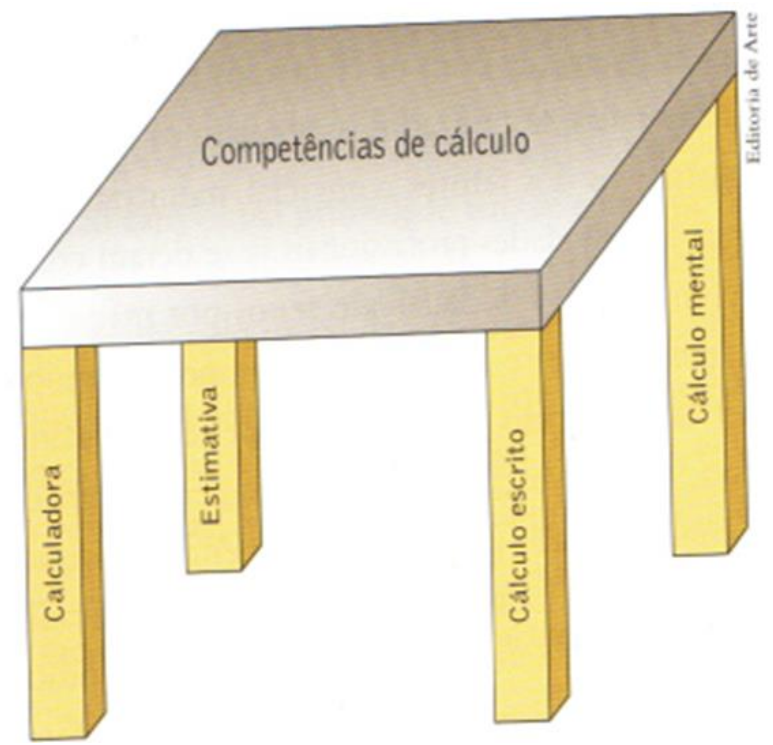

Figura 1: Competências de cálculo (BIGODE e GIMENEZ, 2010, p. 147)

Segundo os Parâmetros Curriculares Nacionais de Matemática para os anos iniciais do Ensino Fundamental, "os diferentes procedimentos e tipos de cálculo relacionam-se e complementam-se" (BRASIL, 1997, p. 75), portanto é importante que o aluno tenha conhecimento do cálculo mental para que este dê suporte para os outros tipos de cálculo, e vice-versa. Dessa forma, o documento corrobora a ideia de Bigode e Gimenez (2010), apontando ser

recomendável que a organização do estudo do cálculo privilegie um trabalho que explore concomitantemente procedimentos de cálculo mental e cálculo escrito, exato e aproximado, de tal forma que 0 aluno possa perceber gradativamente as relações existentes entre eles e com isso aperfeiçoar seus procedimentos pessoais, para tornálos cada vez mais práticos, aproximando-os aos das técnicas usuais. (BRASIL, 1997, p. 75).

Segundo Gomes (2007), os Parâmetros Curriculares Nacionais de Matemática para os anos finais do Ensino Fundamental (BRASIL, 1998) ressaltam a relação entre os diferentes tipos de cálculo, uma vez que o cálculo mental, a estimativa e as aproximações servem de apoio para 0 cálculo escrito. Como já citado anteriormente, até o cálculo escrito se baseia em um encadeamento de operações elementares mentais. Além disso, devido às limitações do cálculo mental, em relação às contas com números de muitos algarismos, faz-se necessário o registro de resultados parciais, utilizando, dessa forma, o procedimento do cálculo escrito. Ribeiro (2005) afirma que, se os professores explorarem na sala de aula as estratégias já utilizadas para efetuar cálculos mentalmente, e apresentarem outras, a turma terá um rendimento melhor nos cálculos escritos.

Em relação às estimativas e ao cálculo mental, os Parâmetros Curriculares Nacionais consideram que "os procedimentos de cálculo por estimativa desenvolvem-se concomitantemente 
aos processos de cálculo mental" (BRASIL, 1997, p. 77). Dessa forma, podemos dizer que 0 cálculo por estimativas pode ser considerado como um processo e uma estratégia para o cálculo mental, portanto, ambos estão diretamente relacionados.

O cálculo mental também é citado na Base Nacional Comum Curricular (BRASIL, 2017a), documento de reorientação curricular referente à Educação Infantil e ao Ensino Fundamental. 0 documento corrobora a relevância e a importância de se trabalhar as quatro modalidades do cálculo matemático, sendo, dessa forma, esperado "que os alunos desenvolvam diferentes estratégias para a obtenção dos resultados, sobretudo por estimativa e cálculo mental, além de algoritmos e uso de calculadoras" (BRASIL, 2017a, p. 266).

Segundo Gomes (2007), na história da Matemática escolar, a prática com o cálculo mental já foi incentivada no passado, no período anterior à Matemática Moderna, sendo justificada pela sua utilidade. Durante o movimento da Matemática Moderna, o cálculo mental foi negligenciado, pois, de acordo com Miorim (1998), citado por Gomes (2007), esse movimento valorizava a abordagem teórica das operações aritméticas e prezava, principalmente, um entendimento das propriedades estruturais dos conjuntos numéricos, não havendo espaço para a valorização nem dos cálculos mentais e de memória, nem das estimativas e aproximações. Atualmente, vivendo um período pós Matemática Moderna, essa prática vem sendo revalorizada e recomendada, não apenas pela dimensão utilitária, mas também pelo seu valor cognitivo.

Gomes (2007) também pesquisou a abordagem dessa temática em livros didáticos. A referida autora apresenta que a recomendação quanto à essa prática tem se refletido nos livros e nos materiais didáticos, os quais têm incentivado o uso do cálculo mental. A autora aponta que a ficha de avaliação das coleções de Matemática do Programa Nacional do Livro Didático de 2006 já possuíam itens específicos destinados a verificar se os livros apresentavam atividades e situações que envolvessem o cálculo mental. O PNLD de 2017, referente ao material didático dos anos finais do Ensino Fundamental, também fez menção ao cálculo mental em sua ficha de avaliação, não obstante, o PNLD de 2018, referente ao Ensino Médio, infelizmente, não cita essa modalidade do cálculo em suas fichas de avaliação.

Uma reflexão importante de ser feita é que, mesmo essa modalidade sendo citada e valorizada nos livros didáticos e outros documentos brasileiros, não significa que realmente exista um trabalho em sala de aula com ela, pois os maiores agentes dentro de sala são os professores, e não os livros didáticos. Professores, sejam eles licenciados em Matemática ou em Pedagogia, muitas vezes não foram preparados para lidar com a prática do cálculo mental em sala de aula. Gonçalves e Freitas (2008) consideram que o cálculo mental parece ser trabalhado apenas no 
Ensino Fundamental e, mesmo assim, ocasionalmente. Os PNC (BRASIL, 1998) mostram que, mesmo que essa modalidade do cálculo seja considerada um conteúdo e um procedimento previsto, ainda existe uma ausência do trabalho com estimativas e cálculo mental nessa etapa da escola básica.

Embora o cálculo mental tenha sido contemplado pela BNCC (BRASIL, 2017a), 0 documento não o menciona como uma prática prevista para $08^{\circ}$ ano e nem $09^{\circ}$ ano do Ensino Fundamental. Dessa maneira, podemos notar que as práticas com o cálculo mental são mais incentivadas durante os anos iniciais do Ensino Fundamental, sendo, muitas vezes, apresentadas junto ao ensino do algoritmo comum.

A versão preliminar da Base Nacional Comum Curricular para o Ensino Médio (BRASIL, 2018), em processo de discussão da comunidade educacional, faz referência a "cálculo", relacionando-o a diversos conteúdos matemáticos, porém, não há referência à sua forma "mental". Os PCN dessa fase do Ensino Básico também não mencionam o cálculo mental.

Atualmente, talvez como reflexo histórico, o cálculo mental é negligenciado durante 0 Ensino Médio, provavelmente devido ao grande volume de conteúdos previstos para essa etapa, e/ou por ser considerado um conteúdo já abordado e aprendido pelos estudantes. Além disso, 0 fato de o ensino da Matemática nos últimos anos da Escola Básica ser focado nas matérias cobradas nos exames para o ensino superior, que, por sua vez, são muito conteudistas, não deixam espaço para essa abordagem. Hoje, esses exames para o ensino superior têm se restringido ao Exame Nacional do Ensino Médio (ENEM), que, por sua vez, é uma prova extensa. Dessa forma, o uso do cálculo mental, que pode ser realizado com mais rapidez e agilidade que 0 cálculo escrito, pode favorecer os alunos que prestam esse Exame e isso deveria ser visto como um bom motivo para que essa prática fosse valorizada no Ensino Médio.

O autor de livros didáticos José Antônio Lopes Bigode, entrevistado por Gentile (2018), entende que "o cálculo mental deve ser um objetivo pedagógico e ser realizado com bastante frequência na classe". Nessa mesma linha de entendimento, Brocardo (2011), considera que "desenvolver o cálculo mental é uma tarefa continuada, a ser levada a cabo em todos os níveis de ensino e de forma sistemática" (p. 10). A autora ainda defende que

em todos os níveis de ensino, faz também sentido propor a descoberta de padrões e regularidades numéricas interessantes e que conduzem a técnicas de cálculo mental que os alunos podem passar a usar. Pode propor-se uma atividade de investigação cuja conclusão permite conhecer uma determinada regra/relação importante para manipular mentalmente um determinado tipo de números e operações. (BROCARDO, 2011, p. 8). 
De acordo com a referida autora, o professor tem um papel fundamental no sentido de desenvolver o cálculo mental dos alunos; ele deve ser capaz de propor tarefas que agucem 0 interesse dos alunos e os estimulem a utilizar o cálculo mental. Brocardo (2011) ainda afirma que uma boa forma de incentivar os professores a disponibilizarem um tempo de suas atividades à prática do cálculo mental é os programas curriculares citarem a importância e as vantagens dessa prática e que especificarem marcos de desenvolvimento para o cálculo mental a cada ano da Escola Básica.

Diante do apresentado, o próximo ponto a ser discutido é a importância e as vantagens de um trabalho com o cálculo mental.

\section{Vantagem e importância}

De acordo com o autor de livro didáticos de Matemática Luis Márcio Imenes, citado por Ribeiro (2005), a maior vantagem do cálculo mental está longe de ser fazer a conta rápida, pois, atualmente, seria prescindível competir com a calculadora. As vantagens do cálculo mental vão muito além da rapidez e agilidade no desenvolvimento do cálculo, no entanto, estas são, sim, vantagens de sua utilização, pois, considerando que, durante a Escola Básica, nem sempre se permite que os estudantes usem calculadora, o cálculo mental, muitas vezes, pode ser efetuado de maneira mais rápida do que o algoritmo tradicional escrito. Sendo uma ferramenta importante para o aluno, o cálculo mental desenvolve técnicas operatórias, todas elas fundamentadas em propriedades matemáticas. Isso possibilita que os alunos compreendam melhor o sistema de numeração utilizado. Para Parra (1996), "o cálculo mental aumenta o conhecimento no campo numérico" (p. 197). Os Parâmetros Curriculares Nacionais para os anos iniciais do Ensino Fundamental ainda orientam que

\footnotetext{
no cálculo mental, a reflexão centra-se no significado dos cálculos intermediários e isso facilita a compreensão das regras do cálculo escrito. O exercício e a sistematização dos procedimentos de cálculo mental, ao longo do tempo, levam-no a ser utilizado como estratégia de controle do cálculo escrito. (BRASIL, 1997, p. 76)
}

Outra vantagem constantemente citada pelos pesquisadores é a relação positiva entre a resolução de problemas e o cálculo mental. Butlen e Pezard (2000), citados por Gonçalves e Freitas (2008), destacam que a prática com o cálculo mental "libera espaço mental" para a resolução de problemas, afirmando que a utilização contínua dessa modalidade de cálculo aumenta a capacidade de iniciativa dos alunos, de maneira que explorem rapidamente diferentes formas de resolver o problema, assim, "o enriquecimento das relações numéricas através do 
cálculo mental facilita para os alunos, frente a uma situação, serem capazes de moldá-la, por antecipação, por reflexão" (PARRA, 1996, p. 195).

Trabalhando sempre com a mente, o cálculo mental estimula a memória, além de desenvolver a qualidade de raciocínio, tanto matemático quanto lógico. Sendo assim, essa modalidade de cálculo aperfeiçoa o senso crítico pessoal. Brocardo (2011) entende que "quando se tem sentido de número e, consequentemente, desenvolvido um bom cálculo mental, conseguese olhar para situações com que nos deparamos de um modo interrogativo" (p. 9). A prática do uso de cálculo mental não é isolada, podendo ser extrapolada em conceitos não matemáticos, por exemplo na capacidade de se elaborar uma frase utilizando os princípios de lógica.

Segundo Araújo e Soares (2002), o cálculo mental está sempre presente em situações reais, por exemplo, no comércio e na economia. Operações com números é uma das partes da Matemática que está presente no cotidiano de todas as pessoas, mesmo fora da escola, portanto considera-se que

os resultados da pesquisa sobre as habilidades matemáticas da população brasileira realizada pelo Indicador de Alfabetismo Funcional (INAF) mostram que as "contas de cabeça", exatas ou aproximadas junto à calculadora são os recursos mais utilizados pelos brasileiros em situações quotidianas. (GOMES, 2007, p.13)

Estimativas e aproximações são processos do cálculo mental e concedem ao aluno a capacidade de antecipar e avaliar resultados de forma aproximada. Para mais, essa modalidade do cálculo também permite ao aluno perceber a existência vários caminhos na resolução de um mesmo problema. Sobre isso, "uma atenção privilegiada ao cálculo mental na escola incentiva os alunos a desenvolverem suas próprias maneiras de enfrentar os desafios que lhes são propostos" (ARAÚJO e SOARES, 2002, p. 35).

\section{Estratégias e propostas}

Iremos, agora, dar um enfoque maior para o "cálculo de cabeça", o cálculo estrategista e pensado, isso pois, visto suas vantagens e já que se fundamenta em propriedades matemáticas, poderá servir para o aluno como um bom instrumento matemático. $O$ trabalho com o cálculo mental e a discussão em sala de aula das estratégias utilizadas para tal podem ser uma boa forma de se trabalhar a identificação e o entendimento das propriedades das operações, as quais, muitas vezes, são negligenciadas por parte dos alunos. De acordo com Ponte, Branco e Matos (2009), "a identificação destas propriedades e a sua generalização desde os primeiros anos de escolaridade 
constituem uma base importante para o pensamento algébrico" (PONTE, BRANCO e MATOS, 2009, p. 28)

Araújo e Soares (2002) consideram a importância de os alunos serem sempre estimulados a elaborar suas próprias estratégias, isso porque, segundo esses autores, diversificar formas de resolver uma operação matemática proporciona uma maior aprendizagem sobre a natureza dos números. Segundo os Parâmetros Curriculares Nacionais, "o cálculo mental apoia-se no fato de que existem diferentes maneiras de calcular e pode-se escolher a que melhor se adapta a uma determinada situação, em função dos números e das operações envolvidas" (BRASIL, 1997, p. 76).

Dessa forma, é importante salientar algumas noções básicas e estratégias para essa modalidade do cálculo, de acordo com o referencial teórico deste artigo, lembrando que o cálculo mental pode ser efetuado de várias formas, portanto, é de extrema importância que cada pessoa utilize o método que ache mais fácil para o cálculo, sendo ele apresentado aqui ou não. Foi optado por se fazer uma apresentação separada, de acordo com as operações.

\section{$\underline{\text { Adição }}$}

Calcular separadamente as unidades das dezenas, das centenas (e assim sucessivamente) e depois unir os resultados parciais. Nesse caso, recorre-se à decomposição decimal de um número, junto à propriedade associativa da adição. Essa estratégia é bem similar à ideia que existe por trás do algoritmo escrito.

- $334+142=(300+30+4+100+40+2)=$

$$
(300+100+30+40+4+2)=(400+70+6)=476
$$

- $194+78=(100+90+4+70+8)=(100+160+12)=(200+72)=272$

$\checkmark$ Completar e compensar. Completa-se uma das parcelas e, na outra, compensa-se com o que foi completado na outra parcela. Nessa estratégia é utilizada a ideia de equivalência das igualdades e, também, utiliza-se a propriedade associativa da adição.

- $36+12=(36+4)+(12-4)=40+8=48$

- $220+95=95+5-5+220=100+115=215$

- $1436+175=(1436+4)+(175-4)=1440+171=(1440+60)+(171-60)$

$$
=1500+111=1611
$$


$\checkmark$ Dobro, dobro mais um, dobro mais dois (e assim sucessivamente). Nessa estratégia também se utiliza a ideia de completar e compensar, no entanto atrelada à multiplicação.

- $53+53=53 \times 2=106$

- $48+49=48+48+1=96+1=97$

- $235+233=235 \times 2-2=470-2=468$

\section{Subtração}

$\checkmark$ Decompor o minuendo e, se precisar, o subtraendo, para depois subtrair. Essa estratégia se baseia na decomposição decimal dos números e utiliza a associatividade da adição de número inteiros juntamente à propriedade distributiva da multiplicação.

- $28-9=20+8-9=11+8=19$

- $97-55=(90+7)-(50+5)=90+7-50-5=40+2=42$

- $132-48=100+30+2-40-8=90-6=80+10-6=84$

- $100-9=(90+10)-9=90+(10-9)=90+1=91$

É importante observar que essa estratégia não precisa ser discutida apenas após o ensino dos números inteiros, pois, caso o aluno ainda não saiba da existência desse conjunto, ele efetuará a conta normalmente, baseando-se nas propriedades dos números naturais.

$\checkmark$ Subtrair por partes de forma a transformar uma subtração difícil em outras mais fáceis. A utilização desse método é bem similar às propriedades existentes no algoritmo escrito.

- $324-71=(324-1)-(71-1)=323-70=303-50=253$

- $1650-390=1350-90=1300-40=1260$

$\checkmark$ Arredondar o minuendo e compensar no subtraendo. As propriedades utilizadas nessa estratégia são as ideias de comparar, completar e compensar da subtração.

- $75-26=75-26+5-5=(75+5)-(26+5)=80-31=50-1=49$

- $890-65=900-75=800+100-75=800+25=825$ 


\section{Multiplicação}

$\checkmark$ Pode-se decompor um ou ambos os fatores pelo sistema decimal e efetuar a conta utilizando a propriedade distributiva da multiplicação.

- $215 \times 8=(200+10+5) \times 8=1600+80+40=1720$

- $1022 \times 43=(1000+20+2) \times(40+3)=40.000+800+80+3.000+60+6=43.946$

$\checkmark$ Pode-se utilizar tabuadas mais fáceis para chegar ao resultado usando a decomposição do número em fatores e a associatividade.

- $232 \times 5=232 \times(10 \div 2)=2320 \div 2=1160$

- $326 \times 6=(326 \times 2) \times 3=652 \times 3=1956$

- $16 \times 21=8 \times 42=4 \times 84=2 \times 168=336$

$\checkmark$ Arredondamento e compensação. Nessa estratégia, utiliza-se a propriedade da distributividade da multiplicação.

- $36 \times 8=36 \times(10-2)=36 \times 10-36 \times 2=360-72=300-12=288$

- $51 \times 19=51 \times 20-51=51 \times 2 \times 10-36=1.020-36=1.000-16=984$

\section{Divisão}

$\checkmark$ Fazer simplificações sucessivas. A ideia por detrás dessa estratégia é achar equivalência de divisões.

- $125 \div 25=(125 \div 5) \div(25 \div 5)=25 \div 5=5$

- $504 \div 36=(504 \div 2) \div(36 \div 2)=252 \div 18=(252 \div 2) \div(18 \div 2)=$

$=126 \div 9=(126 \div 3) \div(9 \div 3)=42 \div 3=14$

Caso não fique claro as simplificações sucessivas dessa forma, pode-se pensar na divisão como uma fração. Vejamos o último exemplo agora de modo fracionário:

$$
\frac{504}{36}=\frac{504 \div 2}{36 \div 2}=\frac{252 \div 2}{18 \div 2}=\frac{126 \div 3}{9 \div 3}=\frac{42 \div 3}{3 \div 3}=14
$$

Deve haver um cuidado em relação à utilização desse método e em relação aos restos dos quocientes, pois, se a divisão não for exata, os restos não serão os mesmos.

$$
\frac{180}{8}=\frac{90}{4}=\frac{45}{2}=22,5\left(22 \text { inteiros } e \frac{1}{2}\right)
$$


No entanto, $180 \div 8=22$ com resto 4 , e $45 \div 2=22$ com resto 1 . A parte decimal do resultado do quociente é sempre em relação ao divisor, por isso o resto do primeiro é metade de 8 e o resto do segundo é metade de 1.

$\checkmark \quad$ Transformar o divisor em uma operação mais conveniente de modo a facilitar as operações. A propriedade utilizada nessa estratégia é a decomposição de um número em outros números por meio da multiplicação e divisão.

- $1005 \div 5=1005 \div(10 \div 2)=1005 \times 2 \div 10=2010 \div 10=201$

- $425 \div 25=425 \div(100 \div 4)=425 \times 4 \div 100=1700 \div 100=17$

$\checkmark \quad$ Pensar quantas vezes o divisor cabe em um dividendo menor e, a partir deste, pensar em um dividendo maior.

- Em $360 \div 18$, o divisor 18 cabe 2 vezes em 36; e 36 cabe 10 vezes em 360. Dessa forma, para cada 10 vezes que 036 coube em 360 , tem-se dois 18 . Então, $360 \div 18=20$

- Em $330 \div 15$, o divisor 15 cabe 2 vezes no 30 . O dividendo é $330=30 \times 11$, tem-se $330 \div$ $15=(30 \div 15) \times 11=22$.

Esse método também contempla as propriedades da multiplicação, visto que, como a divisão é o oposto da multiplicação, dividir por um número é o mesmo que multiplicar pelo inverso dele. Daí, pode-se recorrer à propriedade associatividade da multiplicação. Iremos abordar agora alguns exercícios propostos pelos autores estudados, os quais entendemos ser interessante de serem trabalhados com os alunos, de maneira a incentivá-los a desenvolver o cálculo mental:

(a) Apresentado por Ponte, Branco e Matos (2009, p. 31):

Complete os $\square$ com os símbolos $<$, > ou $=$, de modo a obter afirmações
verdadeiras. Explique seu raciocínio.
\[ \begin{array}{l}38+45 \square 40+43 \\ 40+45 \square 41+45 \\ 39+42 \square 40+43 \\ 35+42 \square 34+40 \\ 38+47 \square 40+43 \\ 52-27 \square 50-25 \\ 55-32 \square 52-32\end{array} \]


O objetivo da questão é explorar o raciocínio de comparação dos alunos, de maneira que eles não efetuem as operações e, sim, analisem as expressões numéricas, e de maneira mental, procurem relações entre os números que as compõem.

(b) Apresentado por Parra (1996, p. 231):

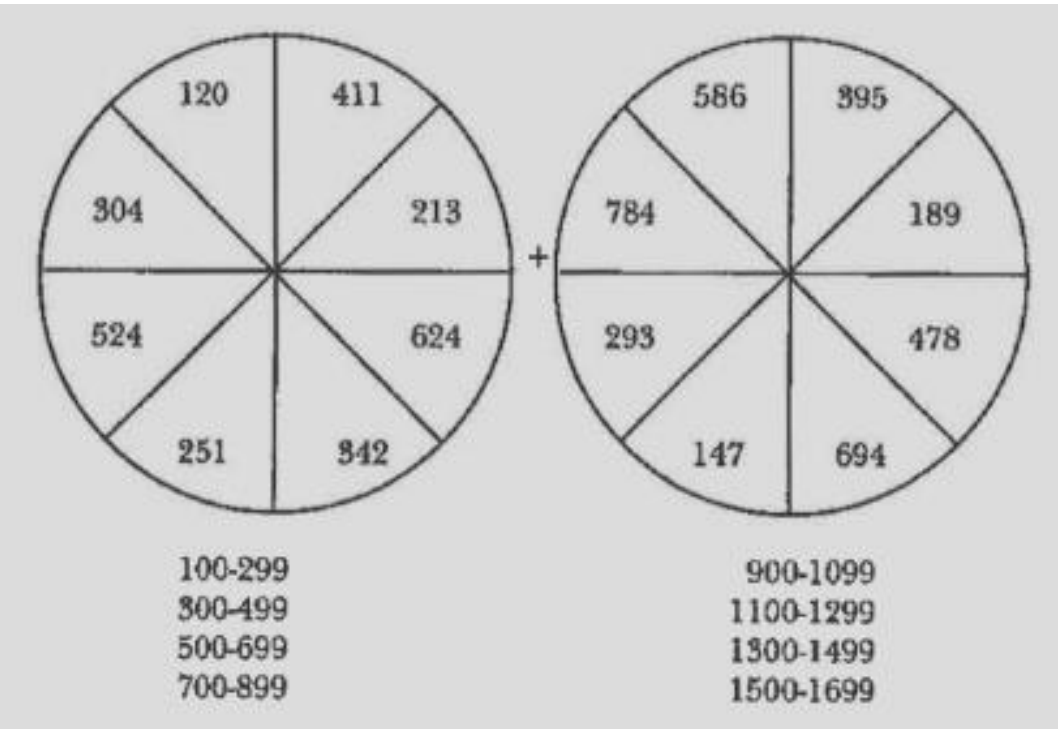

A roleta da estimativa é uma tarefa mais lúdica, na qual é proposta um jogo em que cada participante deve girar a roleta, efetuar mentalmente a operação que julgar mais conveniente (adição ou subtração) e indicar em qual intervalo o resultado se encontra.

(c) Apresentado por Parra (1996, p. 232)

\begin{tabular}{l}
\multicolumn{4}{c}{ Critique e justifique a inexatidão dos } \\
$\begin{array}{lll}\text { (i) } 1913 & \text { para } & 1.547+268 \\
\text { (ii) } 16422 & \text { para } & 27.432-10.510 \\
\text { (iii) } 24624 & \text { para } & 4.230 \times 57 \\
\text { (iv) } 36360 & \text { para } & 630 \times 72 \\
\text { (v) } 107 & \text { para } & 5.421 \div 67 \\
\text { (vi) } 31 & \text { para } & 4.519 \div 15\end{array}$
\end{tabular}

As justificativas esperadas nesses exercícios se baseiem em estratégias e não no resultado da operação apresentada. Por exemplo, em (iii) pode-se concluir errado, pois 2 unidades do número 72 vezes 0 unidades do número 4.230 daria um número com 0 unidades simples, 0 
que não é verdade no número 24.624, apresentado como resposta. Em (v) também pode-se concluir errado, pois $107 \times 67$ daria mais do que $100 \times 67=6.700$, como esse resultado já é maior que o divisor proposto, pode-se concluir que está errada.

\section{Considerações}

Esta pesquisa teve como objetivo entrelaçar diferentes aspectos teóricos sobre o cálculo mental, de acordo com a perspectiva dos autores consultados. Foi explorada a definição desse tipo de cálculo, o que leva ao entendimento que, de certo modo, o cálculo mental pode ser considerado como um cálculo em que se utiliza a mente como instrumento. No entanto, não podemos restringi-lo em apenas isso, pois sendo assim, a ideia de que outros instrumentos não podem ser utilizados seria reforçada. Portanto, é necessário que o cálculo mental seja entendido como um tipo de cálculo em que se utiliza preferivelmente, e não apenas, a mente como o meio em que se realiza as contas.

Foi discutida também a importância do tema e as diferentes vantagens que essa prática pode trazer aos alunos. Dentre as vantagens apresentadas, pode se considerar como mais importante o desenvolvimento do pensamento matemático, uma vez que contribui para uma melhor compreensão das propriedades do sistema de numeração decimal e das operações fundamentais, sendo extremamente vantajoso visto que pode auxiliar na compreensão de conteúdos com maior nível de abstração. Além disso, o desenvolvimento do pensamento lógico e de habilidades para se realizar cálculos também são vantagens consideráveis em relação à prática com o cálculo mental.

A partir do que foi apresentado pelos pesquisadores, como a não valorização do cálculo mental nas escolas, assim como as outras modalidades de cálculo a não ser o escrito, conclui-se que essa estratégia de ensino para cálculos deve ser estimulada em todos os anos do Ensino Fundamental e do Ensino Médio, e não apenas nos anos iniciais da escolarização. Um verdadeiro incentivo à atividade do cálculo mental ocorreria se os programas curriculares brasileiros, assim como a atual Base Nacional Comum Curricular, abordassem esse conteúdo em todos os anos da escola básica, o que infelizmente não acontece.

É importante repensar sobre a função da escola e do professor em relação ao desenvolvimento desta capacidade nos alunos. Sabendo a suma importância do professor em sala de aula, é imprescindível considerar que este seja preparado para abordar e motivar a referida prática, de maneira a incentivar os alunos a praticarem essa modalidade de cálculo. 
Com efeito desta pesquisa bibliográfica, visto a relevância da temática para a formação de professores, o objetivo será dar continuidade a este estudo, de maneira que sejam realizadas, futuramente, pesquisas de campo no Laboratório de Ensino de Matemática (LEM) da Universidade Federal de Minas Gerais, por meio de palestras e oficinas sobre o cálculo mental, voltadas tanto para licenciandos em Matemática quanto em Pedagogia, com o objetivo de discutir sobre essa prática e motivá-los a incentivar tal abordagem nas aulas.

\section{Referências}

ARAUJO, Denise; SOARES, Eduardo. Calculadoras e outras geringonças na escola. Presença Pedagógica, Belo Horizonte, v. 8, n. 47, p. 22-35-27, set./out., 2002.

BIGODE, Antônio José Lopes; GIMENEZ, Joaquim. Metodologia para o ensino da aritmética: competência numérica no cotidiano. São Paulo: FTD, 2010.

BOURDENET Gilles. Le calcul mental. Activités Mathématiques et Scientifiques, Strasbourg, n. 61, p. 5-32, 2007.

BRASIL. Ministério da Educação. Fundo Nacional de Desenvolvimento da Educação. Guia de Livros Didáticos: Matemática - anos finais do Ensino Fundamental. Brasília: MEC/FNDE, 2017b.

BRASIL. Ministério da Educação. Fundo Nacional de Desenvolvimento da Educação. Guia de Livros Didáticos: Matemática - Ensino Médio. Brasília: MEC/FNDE, 2018.

BRASIL. Ministério da Educação. Secretaria de Educação Básica. Base Nacional Comum Curricular. Ensino Médio. Brasília: MEC, 2018.

BRASIL. Ministério da Educação. Secretaria de Educação Básica. Base Nacional Comum Curricular. Brasília: MEC/SEB, 2017a.

BRASIL. Ministério da Educação. Secretaria de Educação Fundamental. Parâmetros Curriculares Nacionais: Matemática. Brasília: MEC/ SEF, 1997.

BRASIL. Ministério da Educação. Secretaria de Educação Fundamental. Parâmetros Curriculares Nacionais: Matemática. Brasília: MEC/SEF, 1998.

BROCARDO, Joana. Uma linha de desenvolvimento do cálculo mental: começando no $1^{\circ}$ ano e continuando até ao $12^{\circ}$ ano. ENCONTRO DE PROFESSORES DE MATEMÁTICA. Actas do PROFMAT2011. Lisboa: Associação de Professores de Matemática (APM), 2011, p. 1-13.

CARVALHO, Raquel. Calcular de cabeça ou com a cabeça? In: ENCONTRO DE PROFESSORES DE MATEMÁTICA. Actas do PROFMAT2011. Lisboa: Associação de Professores de Matemática (APM), 2011, p. 1-8.

FIORENTINI, Dario; LORENZATO, Sérgio. Investigação em Educação Matemática: percursos teóricos e metodológicos. Campinas: Autores Associados, 2006. 
FONTES, Cintia Gomes da. O valor e o papel do cálculo mental nas séries iniciais. 2010. $220 f$. Dissertação (Mestrado em Educação) - Faculdade de Educação. Universidade de São Paulo, São Paulo.

GENTILE, Paola. Cálculo mental: contas de cabeça e sem errar. Nova Escola, São Paulo, 7 mar. 2018.

GOMES, Maria Laura Magalhães. O cálculo mental na história da Matemática escolar brasileira. IN: IX ENCONTRO NACIONAL DE EDUCAÇÃO MATEMÁTICA. Anais do IX ENEM: Diálogos entre a Pesquisa e a Prática Educativa. SBEM, 2007, p. 1-16.

GONÇALVES, Maria José Santana Vieira; FREITAS, José Luiz Magalhães. O cálculo mental como ferramenta e objeto durante o estudo de proporcionalidade por alunos do $7^{\circ}$ ano do Ensino Fundamental. In: XII ENCONTRO BRASILEIRO DE ESTUDANTES DE PÓS-GRADUAÇÃO EM EDUCAÇÃO MATEMÁTICA. Anais do XII EBRAPEM. Rio Claro, UNESP, 2008, p. 1-10.

GUIMARÃES, Sheila Denize; FREITAS, José Luiz Magalhães de. Um olhar sobre o papel do cálculo mental para a aprendizagem de conceitos matemáticos nos anos iniciais do Ensino Fundamental. IN: IX ENCONTRO NACIONAL DE EDUCAÇÃO MATEMÁTICA. Anais do IX ENEM: Diálogos entre a Pesquisa e a Prática Educativa. SBEM, 2007, p. 1-11.

HOLANDA, Aurélio Buarque de. Dicionário Aurélio da Língua Portuguesa. 5. ed. Curitiba: Positivo, 2014.

PARRA, Cecilia. Cálculo mental na escola primária. In: PARRA, Cecilia; SAIZ, Irma. Didática da Matemática: reflexões psicopedagógicas. Tradução de Juan Acuña Llorens. Porto Alegre: Artmed, 1996, p.186-235.

PONTE, João Pedro da; BRANCO, Neusa; MATOS, Ana. Álgebra no ensino básico. Lisboa: DGIDC, 2009.

RIBEIRO, Raquel. Cálculo mental: quanto mais diversos os caminhos, melhor. Nova Escola, São Paulo, 1 abr. 2005. 